\title{
Historia natural cuantitativa de una relación parásito-hospedero: el sistema Tristerix-cactáceas en Chile semiárido
}

\author{
Quantitative natural history of a host-parasite relationship: the Tristerix-cactus system \\ in semiarid Chile
}

\author{
RODRIGO MEDEL, CAREZZA BOTTO-MAHAN, CECILIA SMITH-RAMÍREZ, MARCO A. MÉNDEZ, CARMEN G. OSSA, \\ LUCIANO CAPUTO \& WILFREDO L. GONZÁLES
}

Departamento de Ciencias Ecológicas, Facultad de Ciencias, Universidad de Chile, Casilla 653, Santiago, Chile; e-mail: rmedel@abello.dic.uchile.cl

\begin{abstract}
RESUMEN
Presentamos información cuantitativa de la historia natural de la relación parásito-hospedero constituida por el muérdago holoparásito Tristerix aphyllus (Loranthaceae) y sus hospederos cactáceas. Más específicamente, indagamos en los determinantes históricos y biogeográficos de la interacción y cuantificamos la autoecología de la biología floral, polinización, dispersión y parasitismo en este sistema. El impacto del parasitismo sobre la evolución de sistemas defensivos en las cactáceas hospederas es considerado tanto a nivel intraespecífico como interespecífico, tomando en cuenta el potencial para selección mediada por parásitos y la estructura geográfica de la interacción. Finalmente, sugerimos futuras avenidas de investigación en este sistema que incluyen los tópicos de: (i) evolución de la virulencia, (ii) estructuración de la interacción en mosaico geográfico y, (iii) pruebas históricas de adaptación. Estos aspectos permitirán adquirir un mayor conocimiento de la sutileza ecológica y de la evolución de esta especial interacción en los sistemas naturales de Chile semiárido.
\end{abstract}

Palabras clave: Loranthaceae, Cactaceae, parasitismo, estrategias defensivas, mosaico geográfico, muérdago, coevolución.

\section{ABSTRACT}

We present quantitative information on the natural history of a host-parasite interaction that consists on the holoparasitic mistletoe Tristerix aphyllus (Loranthaceae) and its cacti host species. More specifically, we inquire into the historical and biogeographical setting of the relationship, and quantify the autoecology of the floral biology, pollination, seed dispersal, and parasitism of the system. The impact of the mistletoe on the evolution of defense systems is evaluated both at intraspecific and interspecific levels through consideration of the potential for parasite-mediated selection and the geographical structure of the host-parasite interaction. Finally, we suggest prospective lines of research which include aspects related to: (i) the evolution of virulence, (ii) the geographic structure of the interaction, and (iii) the historical evidence for adaptation. These topics will allow us to gain a better understanding of the ecological subtlety and evolution of this singular interaction in semiarid Chile.

Key words: Loranthaceae, Cactaceae, parasitism, defense strategies, geographic mosaic, mistletoe, coevolution.

\section{INTRODUCCIÓN}

Las plantas parásitas representan aproximadamente un $1 \%$ del total de las especies angiospermas, con más de 3.000 especies distribuidas en 16 familias (Kuijt 1969, Musselman \& Press 1995). La mayor parte de los estudios efectuados sobre plantas parásitas se han focalizado en la ecología de la frugivoría y dispersión de las semillas (e.g., Reid 1986, Herrera 1988, Reid 1989, 1991, Murphy et al. 1993, Larson 1996) y sólo recientemente se ha incorporado la perspectiva parásito-hospedero (e.g., Watkinson \& Gibson 1988, Dobson \& Crawley 1994, Matthies 1995, Medel et al. 1995, Martínez del Río et al. 1996,
Marvier 1996, Matthies 1996). Sorprendentemente pocos estudios han evaluado el impacto de las plantas parásitas sobre la adecuación biológica de sus hospederos en poblaciones naturales (e.g., Gómez 1994, Gomes \& Fernandes 1994, Silva \& Martínez del Río 1996, Medel 2000), y por consiguiente el impacto evolutivo del parasitismo sobre sus hospederos plantas es prácticamente desconocido (Dobson \& Crawley 1994, Norton \& Carpenter 1998). A pesar de su importancia evidente para estudios de coevolución parásito-hospedero, los estudios sobre la evolución de las plantas parásitas en su asociación con sus plantas hospederas son prácticamente inexistentes (e.g., Medel 2000, Mutikainen et al. 2000). 
El objetivo de este trabajo es presentar información cuantitativa de la historia natural y autoecología de Tristerix aphyllus (Miers ex DC.) Tiegh. ex Barlow et Wiens, un muérdago (Familia: Loranthaceae) endémico a la región árida y semiárida de Chile que parasita únicamente a especies de la familia Cactaceae (Follman \& Mahú 1964, Kuijt 1969, 1988). La singular forma de vida del muérdago proporciona un atractivo material para indagar acerca de los determinantes ecológicos y consecuencias evolutivas de su estrecha relación con hospederos cactáceas. Por ejemplo, T. aphyllus es la única especie de la familia que carece enteramente de hojas. Esta singular característica, útil para evitar desecación en ambientes áridos, se ha sugerido que representa una adaptación a la vida en zonas áridas y a su modo de vida holoparasítico de cactáceas (Mauseth 1985, 1991). La porción vegetativa del muérdago consiste en un endófito al interior de la cactácea y, a diferencia de la mayoría de los muérdagos hemiparásitos que se adhieren a los tejidos xilemáticos de sus plantas hospederas, T. aphyllus parasita los vasos floemáticos de los cactos (Mauseth et al. 1984, 1985), siendo el componente reproductivo del muérdago la única porción del parásito que emerge de los tejidos de sus hospederos. En este trabajo se describirán una serie de estadígrafos de la interacción planta parásita - planta hospedera, ilustrando sus componentes más importantes mediante el sistema modelo Tristerix aphyllus Echinopsis chilensis (Echinopsis chiloensis [Co1la] Friedrich et G.D. Rowley). Los estadígrafos fueron obtenidos de un estudio continuado de 8 años efectuado en la Reserva Nacional Las Chinchillas, Illapel, IV Región, Chile (31 ${ }^{\circ} 30^{\prime} \mathrm{S}, 71^{\circ}$ 06' O). Asimismo, se indagará sobre el potencial valor adaptativo de sistemas de defensa de las cactáceas, enfatizando la necesidad de incorporar perspectivas locales y regionales al estudio de la adaptación.

\section{ANTECEDENTES HISTÓRICOS DE LA INTERACCIÓN}

La familia de las plantas parásitas Loranthaceae consta de aproximadamente 900 especies distribuidas en 65 géneros. La familia se diversificó en climas templados de altas latitudes, probablemente en bosques cerrados de Gondwanalandia en el Cretácico medio hace 70 millones de años (Barlow 1983). Desde entonces, formas secundarias han avanzado y se han diferenciado hacia los trópicos en Sudamérica, ocurriendo un avance importante de formas Australasianas durante el Eoceno (58-36 106 años) (Villagrán \& Hinojosa
1997). El género Tristerix, consta de 11 especies las cuales están enteramente circunscritas a Sudamérica donde habitan desde sectores adyacentes a la Cordillera de Los Andes en Chile y Argentina hasta el sub-páramo de Colombia y Ecuador (Fig. 1) (Kuijt 1988). Se ha sugerido que el grupo más afín de este género se encontraría en Nueva Zelandia (Barlow 1983). Sin embargo, esta proposición se ha cuestionado indicándose que Tristerix presentaría una mayor afininidad con otros grupos Sudamericanos más que Neozelandeses (Kuijt 1988). Recientes proposiciones de relaciones filogenéticas para plantas parásitas han sugerido que la forma de vida holoparásita es una condición derivada, siendo el estilo de vida hemiparásito (parásitos con hojas) el ancestral en el grupo (de Pamphilis 1995). Es probable que la ausencia de hojas en $T$. aphyllus constituya un carácter derivado y probablemente adaptativo al parasitismo sobre cactáceas.

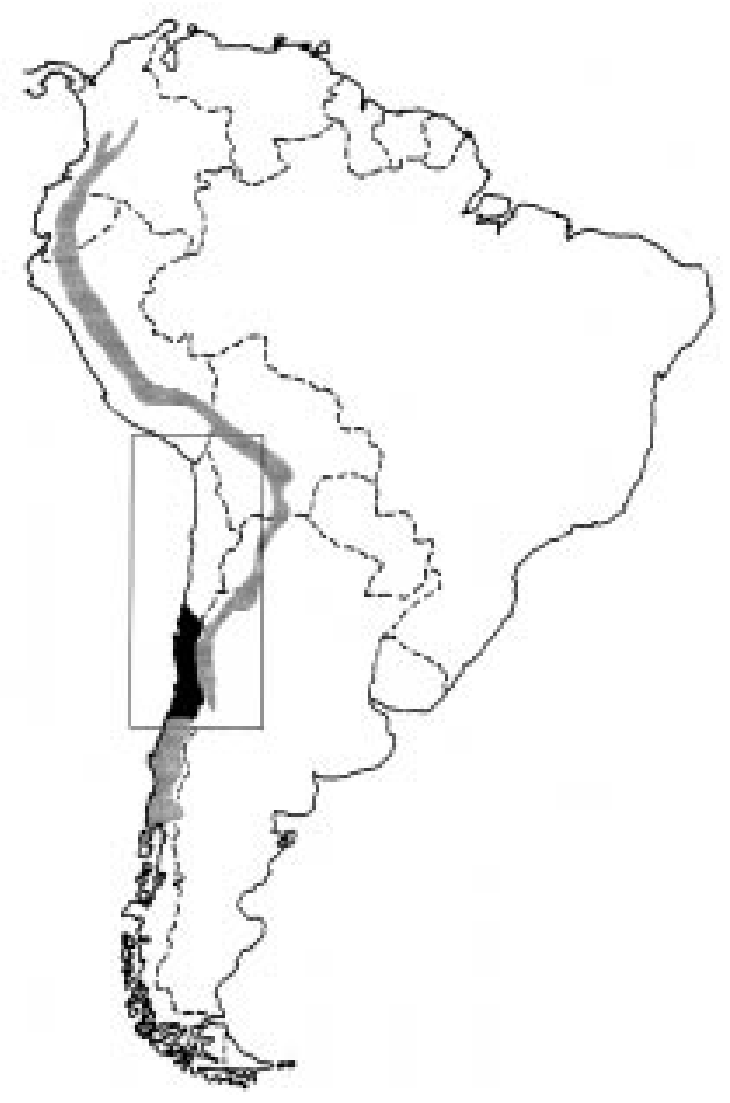

Fig. 1: Mapa de la distribución geográfica de Tristerix (gris). En negro se destaca la distribución de Tristerix aphyllus. Modificado de Kuijt (1988).

Map of the geographical distribution of Tristerix (grey). The distribution of T. aphyllus is depicted in black. Modified from Kuijt (1988). 
Tristerix aphyllus es una especie endémica a Chile con un rango de distribución que va desde los 27 a $\operatorname{los} 34,5^{\circ} \mathrm{S}$. La especie parasita principalmente cactos columnares de los géneros Echinopsis (ex Trichocereus) y Eulychnia (Follman \& Mahú 1964), cuyas especies habitan en ambientes áridos y semiáridos de Chile. Recientes estudios sugieren que los elementos xéricos existentes en el centronorte de Chile, probablemente tienen su fuente en bajas latitudes desde donde habrían emigrado hacia Chile central a partir del Mioceno medio (14,7 $10^{6}$ años), coincidente con el inicio de la formación del desierto de Atacama (Alpers \& Brimhall 1988). Sin embargo, fue probablemente durante el Plioceno, hace no más de $510^{6}$ años, que las especies pudieron haber adoptado su configuración distribucional actual debido a la intensa aridización asociada a los cambios climáticos producto del levantamiento final de la Cordillera de Los Andes y al inicio de los fenómenos de surgencia de la corriente de Humboldt (Solbrig 1976, Solbrig et al. 1977, Kalin-Arroyo et al. 1995). Es posible que la intensificación de las condiciones de aridez en aquel período haya determinado el establecimiento y la diversificación de los géneros de cactáceas en el centro-norte de Chile. La temprana diversificación de Loranthaceae en el Cretácico medio y su subsecuente avance y diferenciación hacia el Ecuador a partir del Eoceno, sugiere la presencia de una flora hemiparásita con anterioridad al establecimiento de su comunidad de hospedadores cactáceas en el centro-norte de Chile durante el Plioceno. Sobre la base de esta información es posible plan- tear la hipótesis que la interacción Tristerixcactáceas no se debiera extender más allá de $510^{6}$ años, período desde el cual Tristerix probablemente ha extendido el rango de hospederos parasitados incluyendo un creciente número de especies de los géneros Echinopsis y Eulychnia.

\section{BIOLOGÍA FLORAL Y POLINIZACIÓN DE TRISTERIX APHYLLUS}

El período de floración de T. aphyllus se extiende desde marzo a agosto, aunque algunas yemas que se generan durante el invierno permiten encontrar flores aisladas durante septiembre y octubre. Las flores se abren secuencialmente, observándose un máximo de flores abiertas en abril-junio. Las flores son tubulares y formadas por cuatro tépalos de color rojo, los cuales están fusionados en su primera mitad. El estilo y los cuatro estambres, todos de color amarillo, sobresalen del tubo floral. Las flores están insertas en una inflorescencia de tipo panícula compuesta, la cual se compone de un pedúnculo basal ramificado de $61,0 \mathrm{~mm}$ de longitud (Tabla 1). El número total de flores por inflorescencia es variable, pudiendo en los meses de máxima floración alcanzar hasta un máximo de 3.000 flores. Cada flor presenta un solo óvulo. Los estadígrafos de tendencia central y dispersión de los caracteres morfológicos de las flores de T. aphyllus, así como de las características del néctar producido son indicados en la Tabla 1.

TABLA 1

Estadística descriptiva de la inflorescencia y de la función floral de Tristerix aphyllus sobre Echinopsis chilensis en la Reserva Nacional Las Chinchillas, Aucó, IV Región

Descriptive statistics of the inflorescence and floral function of T. aphyllus on E. chilensis

\begin{tabular}{|c|c|c|c|}
\hline Rasgo & Promedio (DE) & Rango & $\mathrm{n}$ \\
\hline \multicolumn{4}{|l|}{ Inflorescencia } \\
\hline Angulo de exposición $\left({ }^{\circ}\right)$ & $132,6(49,9)$ & $36,0-267,0$ & 118 \\
\hline Longitud pedúnculo basal (mm) & $61,0(30,5)$ & $4,6-290,8$ & 455 \\
\hline Número de pedúnculos laterales & $24,2(19,2)$ & $1-75$ & 560 \\
\hline \multicolumn{4}{|l|}{ Flor } \\
\hline Longitud pedicelo (mm) & $5,9(1,6)$ & $0,1-10,6$ & 786 \\
\hline Longitud ovario $(\mathrm{mm})$ & $4,0(0,6)$ & $2,1-7,7$ & 866 \\
\hline Longitud estilo (mm) & $41,2(4,4)$ & $2,2-36,5$ & 866 \\
\hline Longitud estambre (mm) & $39,6(4,5)$ & $19,4-71,4$ & 860 \\
\hline Ancho flor $(\mathrm{mm})$ & $3,5(0,5)$ & $2,1-5,6$ & 847 \\
\hline Ancho antera $(\mathrm{mm})$ & $1,4(0,3)$ & $1,1-1,9$ & 853 \\
\hline \multicolumn{4}{|l|}{ Néctar } \\
\hline Volumen en $17 \mathrm{~h}(\mathrm{ml})$ & $11,4(9,6)$ & $0,3-71,3$ & 854 \\
\hline Concentración (\% peso/peso) & $19,5(3,2)$ & $4,0-30,0$ & 564 \\
\hline
\end{tabular}


Ensayos de polinización manual con polen alógeno (de una flor de otro individuo), autopolinización manual (de la misma flor), automática, apomixis y control revelaron que $T$. aphyllus es parcialmente autocompatible y agamospérmica en un $15 \%$ (Tabla 2). Algunas especies de mariposas (género Phoebis) y dípteros visitan esporádicamente las flores de T. aphyllus en busca de néctar, pero no contactan las anteras con los estigmas de la flor. Hormigas de los géneros Camponotus ( $C$. morosus), Solenopsis ( $S$. gayi) y Conomyrma ( $C$. goestchi) visitan a menudo las flores, "robando" néctar a través de incisiones en la cámara.

La coloración roja de las flores evidencia la ocurrencia de polinización a través de aves. Se ha demostrado en terreno que la polinización ocurre exclusivamente a través de la visita del picaflor Sephanoides galeritus, especie migratoria y residente de invierno en el centro-norte de Chile. La mayor abundancia de $S$. galeritus en el norte de Chile, ocurre entre mayo - julio, coincidente con la floración masiva de T. aphyllus. En esta fecha, los picaflores exhiben una alta densidad (2-4 ind $\mathrm{ha}^{-1}$ ), lo cual da cuenta de la alta tasa de visitas sobre T. aphyllus. Observaciones efectuadas durante $66 \mathrm{~h}$ en mayo 1995 revelaron que cada inflorescencia del muérdago es visitada a una tasa de 1,79 visitas $\mathrm{h}^{-1}$. Una vez visitada la inflorescencia, el picaflor contacta el interior de 29,2 flores en promedio, lo cual representa un $28 \%$ del total de las flores abiertas en cada inflorescencia (número total de flores contactadas $=1.123$ ). Los picaflores establecen territorios y rutas definidas que incluyen varios individuos de $T$. aphyllus ubicados en distintos hospederos. Sólo en 12 ocasiones (correspondiendo aproximadamente al 0,4 $\%$ del total de visitas) se repitieron las visitas a las mismas flores en períodos de observación de 30 min. Los territorios florales son defendidos activamente, registrándose un $15 \%$ de encuentros agresivos intraespecíficos en el período de observación señalado.

\section{DISPERSIÓN DE SEMILLAS DE TRISTERIX APHYLLUS}

La estación de dispersión de T. aphyllus se extiende desde fines de marzo a fines de noviembre, ocurriendo la máxima producción de frutos entre julio - septiembre. Durante este período el 70,6 \pm $0,12 \%$ de las flores de cada inflorescencia produce frutos (media $\pm \mathrm{DE}, \mathrm{n}=14$, rango $=45,2-$ $83,3)$. El área de la infructescencia es de $306,5 \pm$ $302,5 \mathrm{~cm}^{2}(\mathrm{n}=72$, rango $=62,8-1.609,3)$, con una densidad de $0,806 \pm 0,266$ frutos $\mathrm{cm}^{-2}$ (rango $=$ $0,302-1,431, \mathrm{n}=59$ infructescencias). Los frutos son seudobayas alargadas de color verde - rojo opaco en estados de inmadurez, tornando a formas esféricas blanco translúcidas al madurar. Los frutos maduros poseen un diámetro de 9,14 $\pm 0,71$ $\mathrm{mm}$ y un peso de 0,369 $\pm 0,097 \mathrm{~g}$ (media $\pm \mathrm{DE}, \mathrm{n}$ $=375$ ). Una vez secos, los frutos pesan 0,064 \pm $0,018 \mathrm{~g}$. La semilla es elongada con un eje mayor de 7,08 $\pm 0,72 \mathrm{~mm}$ y un eje menor de 2,58 $\pm 0,43$ $\mathrm{mm}(\mathrm{n}=375)$. Las semillas, una vez dispersadas y por efecto de la deshidratación, se recubren enteramente por una viscina pegajosa que facilita su adhesión a cualquier superficie.

Los frutos de T. aphyllus son consumidos, aunque no dispersados, por roedores (Abrothrix olivaceus), larvas de lepidópteros y marginalmente por reptiles (Liolaemus sp. y Callopistes palluma) (Smith-Ramírez 1999). Observaciones efectuadas desde 1994 en diferentes poblaciones de Chile central indican que, al igual que los muérdagos de otras latitudes, T. aphyllus es dispersado exclusivamente por aves (e.g., Reid 1986, 1991, pero véase Amico \& Aizen 2000). El único ave que participa en la remoción y dispersión de las semillas es el mímido Mimus thenca (Mimidae), especie cuyo límite norte de distribución coincide con las poblaciones más nortinas del muérdago (Araya \& Millie 1986). Mimus thenca tiende a defender territorios definidos y a favorecer el uso de algunos cactos como perchas, depositando mayoritariamente las semillas de T. aphyllus en

TABLA 2

Proporción de frutos-flor de Tristerix aphyllus en distintos ensayos de polinización, durante el año 1995 en la Reserva Nacional Las Chinchillas, Aucó, IV Región

Fruit set of T. aphyllus in different pollination assays during 1995

\begin{tabular}{lcc}
\hline Tipo de cruzamiento & $\begin{array}{c}\text { Número de individuos } \\
\text { (total de flores ensayadas) }\end{array}$ & $\begin{array}{c}\text { Frutos producidos/total } \\
\text { de flores ensayadas }\end{array}$ \\
\hline Apomixis & $45(60)$ & 0,15 \\
Automática & $16(16)$ & 0,50 \\
Alógeno manual & $16(16)$ & 0,30 \\
Automático manual & $16(20)$ & 0,54 \\
Abierta & $29(290)$ & 0,47 \\
\hline
\end{tabular}


cactos previamente infectados y generando, de este modo, distribuciones altamente agrupadas del muérdago (Martínez del Río et al. 1995, 1996). Mimus thenca ingiere los frutos maduros por completo, a una tasa de $1,33 \pm 0,97$ semillas día ${ }^{-1}$ infructescencia-1 $(n=408)$, y defeca las semillas, no más de tres a la vez, sobre rocas, arbustos y la mayor parte de las veces sobre los propios cactos.

Las semillas de $T$. aphyllus germinan durante las primeras $24 \mathrm{~h}$ en un $87 \%$ de las veces y en un $91 \%$ transcurrida la primera semana. Pasada la primera semana la radícula crece a una tasa sigmoidal, exhibiendo rangos de variación en longitud que van desde 14 a $91 \mathrm{~mm}$ a los 43 días de crecimiento (Martínez del Río et al. 1995). No obstante lo anterior, la radícula puede crecer hasta 56 días después de defecada la semilla, elongándose hasta un máximo de $137 \mathrm{~mm}$ en condiciones naturales. Una vez contactada la epidermis del cacto por la radícula, se establece una placa haustorial desde la cual varios filamentos penetran el parénquima (Mauseth et al. 1984, 1985). Es en este tejido donde T. aphyllus crece vegetativamente hasta la nueva emergencia de la porción reproductiva por las areolas de la cactácea al año siguiente (Botto-Mahan et al. 2000). La porción reproductiva de $T$. aphyllus se encuentra inmersa en la matriz de espinas de E. chilensis y emerge preferentemente en la porción SE de la columna infectada (Botto-Mahan et al. 2000, Tabla 1$)$.

\section{SISTEMAS DEFENSIVOS DE LOS HOSPEDEROS}

\section{Evidencias a nivel poblacional}

Echinopsis chilensis es una cactácea columnar que habita en las laderas de exposición ecuatorial en el centro-norte de Chile. Su estación reproductiva se extiende desde octubre hasta mediados de febrero, dependiendo del año. El número de costillas de E. chilensis presenta un rango entre 9 y 20 , sugiriendo que este carácter no debiera ser considerado diagnóstico para la determinación de la especie. Asimismo, no existen diferencias significativas respecto a este rasgo entre individuos infectados y no infectados por T. aphyllus (Tabla 3). E. chilensis presenta una gran variación en la longitud de las espinas apicales entre poblaciones (Rundel 1976, Hoffmann 1986), probablemente como consecuencia de variación geográfica en la prevalencia e intensidad del parasitismo (Medel 2000). Las areolas de la cactácea presentan 1 a 2 espinas principales esclerificadas que se proyectan hacia el exterior y de 8 a 12 espinas laterales cortas que se disponen en múltiples direcciones.

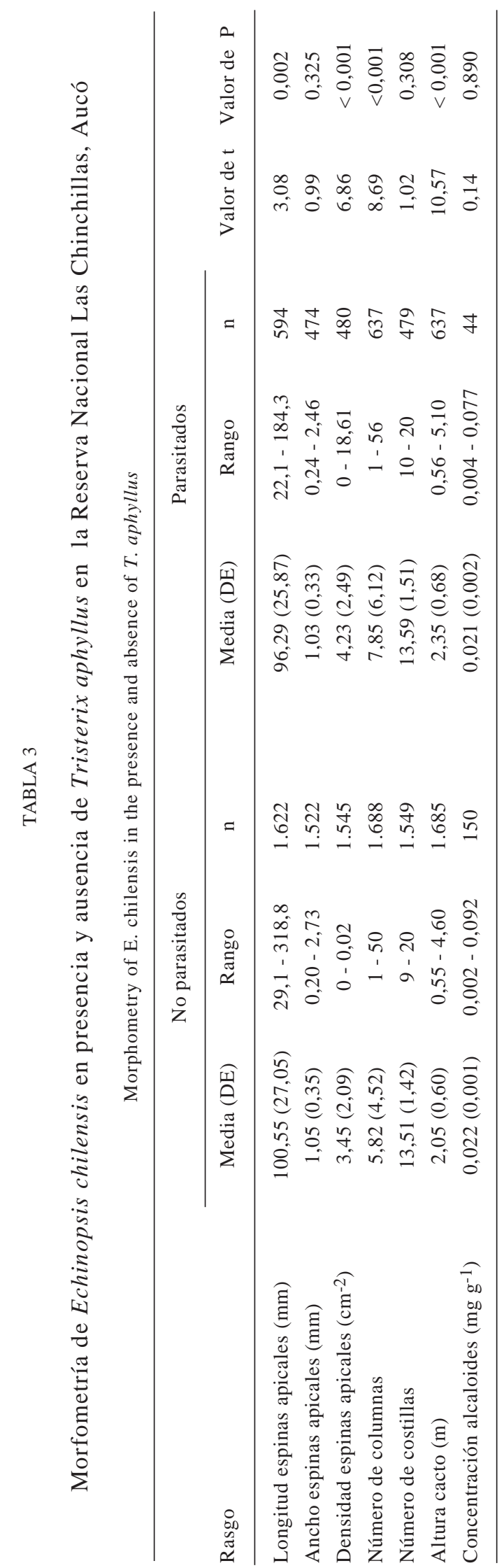


Estudios previos han indicado que la longitud de las espinas apicales afecta la probabilidad de los cactos de llegar a ser parasitados por T. aphyllus. De hecho, los individuos de E. chilensis que no son visitados por $M$. thenca tienen espinas en promedio $1,29 \mathrm{~cm}$ más largas que los individuos visitados (Medel 2000). De igual forma, los individuos de E. chilensis que no reciben semillas poseen espinas $0,84 \mathrm{~cm}$ más largas que los individuos que reciben infección (Medel 2000). Estas evidencias indican que el carácter longitud de espinas constituye una primera barrera contra la infección por T. aphyllus. Recientes análisis, sin embargo, que incluyen los datos agrupados de individuos de un mayor número de poblaciones (10 poblaciones, $\mathrm{n}=2.216$ individuos) han revelado una mayor diferencia en la longitud de espinas entre los ejemplares no parasitados y parasitados $(4,26 \mathrm{~cm}$ de diferencia en promedio, Tabla 3). Es posible que la variación observada a escala local sea absorvida por una mayor variación a escala metapoblacional respecto a este rasgo defensivo. El ancho de las mismas espinas, sin embargo, no pareciera ser de importancia en relación al status de parasitismo (Tabla 3). La densidad de las espinas apicales es mayor en la fracción parasitada que en la no parasitada (Tabla 3), lo cual sugiere que espinas más densamente dispuestas pueden favorecer mas que impedir el uso de los cactos como percha por el vector $M$. thenca. Es posible sugerir la existencia de un compromiso entre la densidad y la longitud de las espinas apicales de las cactáceas. Los individuos parasitados poseen en promedio 2,03 columnas más que los ejemplares no parasitados (Tabla 3), lo cual se ha sugerido que representa un mecanismo de tolerancia asociado a la pérdida de dominancia apical de las columnas una vez eliminado el módulo parasitado (Medel 2001). Los cactos parasitados son $0,3 \mathrm{~m}$ más altos en promedio que los individuos no parasitados. Este hecho fue notado por Martínez del Río et al. (1995), quienes asociaron este fenómeno a la preferencia de $M$. thenca por perchas de mayor altura con la consiguiente infección de las cactáceas más altas. Es posible que el comportamiento territorial del ave sea un elemento crítico que determina la infección y reinfección en este sistema.

El estatus de infección de los individuos de $E$. chilensis es variable a lo largo del tiempo. Una población de 223 individuos marcada en 1994 experimentó diferentes probabilidades de cambio de un status de infección a otro (i.e., de status no parasitado $\rightarrow$ parasitado y viceversa). Un muestreo efectuado en el año 2000 reveló que el $26 \%$ de los individuos inicialmente no parasitados en 1994 adquirieron la infección a lo largo de los 6 años, en tanto que un $8,1 \%$ se recuperó del parasitismo externo mediante la pérdida de los módulos parasitados (Fig. 2).

La presencia de $T$. aphyllus reduce significativamente los distintos componentes de la adecuación Darwiniana de E. chilensis. Respecto al componente fecundidad, la producción de frutos es reducida en un 58,8 \%, la producción de semillas por fruto en un 71,3\%, y la producción total de semillas por cacto en un $67,1 \%$, en comparación a los valores observados en ausencia de $T$. aphyllus (Fig. 3). En relación al componente sobrevivencia, los resultados indican que los ejemplares parasitados sufren una mortalidad un orden de magnitud mayor que los individuos no parasitados (Fig. 2).

Como las plantas pueden exhibir líneas de defensas físicas (tricomas, espinas) y/o químicas (metabolitos secundarios) contra herbívoros o parásitos, la mantención de diversas líneas defensivas podría implicar la existencia de compromisos fenotípicos o genotípicos entre dichos caracteres (Herms \& Mattson 1992, Fineblum \& Rausher 1995). El rol de diversas familias de metabolitos secundarios como defensa contra herbivoría ha sido ampliamente estudiado (ver Rosenthal \& Berenbaum 1991). En particular, los alcaloides, un grupo importante de metabolitos secundarios presente en diversas familias vegetales, incluyendo a las cactáceas (Harborne \& Baxter 1993), han sido asociados a defensa contra parásitos herbívoros (Hartmann 1991). En este contexto, se ha comenzado a indagar el patrón de distribución de alcaloides entre estructuras

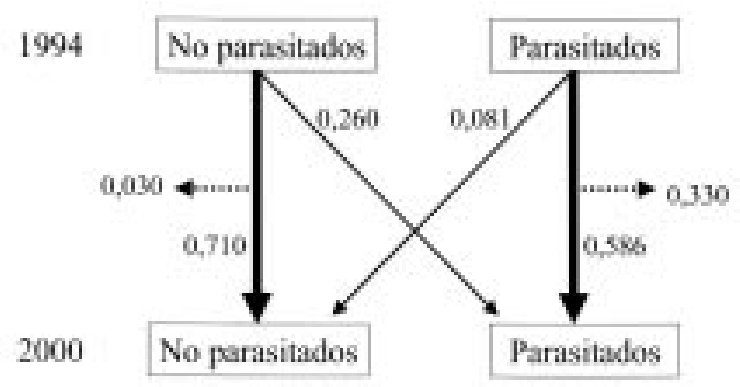

Fig. 2: Diagrama de probabilidades observadas de mantención y cambio de estatus de parasitismo experimentado por Echinopsis chilensis entre los años 1994-2000 en la Reserva Nacional Las Chinchillas, Aucó, IV Región. Las líneas segmentadas indican la probabilidad de mortalidad experimentada por los cactos en el lapso de 6 años.

Diagram of the observed probabilities of maintenance and change of status of parasitism experienced by E. chilensis between 1994-2000. Dashed lines indicate the mortality suffered by cacti in the lapse of 6 years. 

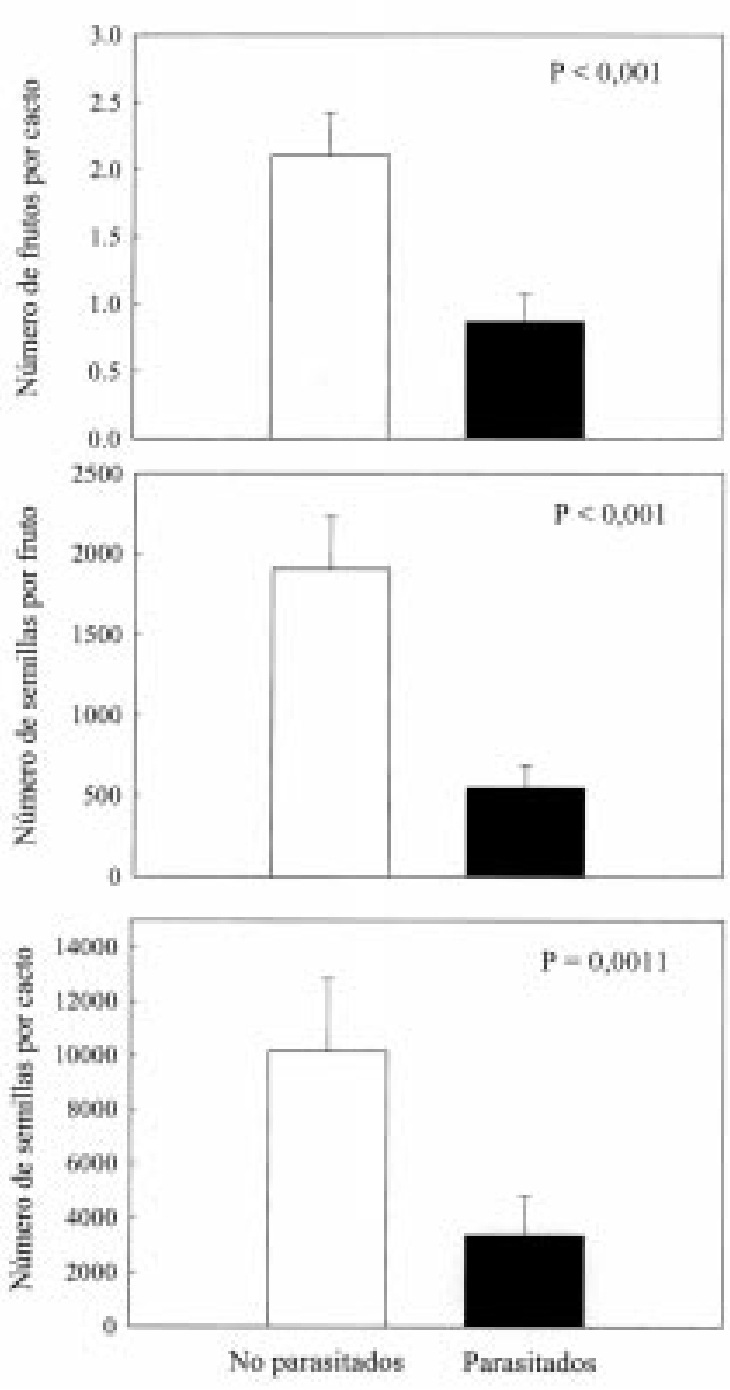

Fig. 3: Impacto promedio de Tristerix aphyllus sobre tres componentes de la fecundidad de Echinopsis chilensis en la Reserva Nacional Las Chinchillas, Aucó, IV Región. Grados de libertad $=216$ en todos los casos. Barras indican +1 EE.

Mean impact of parasitism of T. aphyllus upon three fecundity components of E. chilensis. Degress of freedom $=216$ in every case. Bars indicate $+1 \mathrm{SE}$.

vegetativas y reproductivas de E. chilensis, y su potencial rol defensivo contra T. aphyllus. Resultados preliminares indican ausencia de diferencias en la concentración de alcaloides totales en el parénquima de las cactáceas parasitadas y no parasitadas (Tabla 3 ), sugiriendo que no existirían mecanismos de defensa química constitutiva en el tejido vegetativo de los individuos. No obstante lo anterior, análisis pareados han revelado diferencias significativas en la concentración de alcaloides entre el tejido vegetativo de la cactácea y el tejido reproductivo (frutos) (media \pm EE, vegetativo: $0,0170 \pm 0,003$; reproductivo: $0,058 \pm 0,006, \mathrm{n}=10, \mathrm{z}=2,80, \mathrm{P}=0,005)$. Estos resultados sugieren la existencia de una mayor asignación a defensa química en el componente reproductivo, probablemente como respuesta al parasitismo de los frutos de los cactos por insectos lepidópteros.

\section{Evidencias a nivel de especie}

Actualmente la interacción T. aphyllus-cactáceas ocurre en la zona centro-norte de Chile, entre los 27 y $\operatorname{los} 34,5^{\circ} \mathrm{S}$, donde parasita diversas especies de los géneros Echinopsis (E. chilensis, E. coquimbana, E. litoralis, E. skottsbergii, y E. spinibarbis) y Eulychnia (E. acida, E. breviflora y E. castanea) con combinaciones de hospederos variables entre localidades (Fig. 4). Como en cada género existen especies no parasitadas (i.e., aquellas situadas a una latitud menor $27^{\circ} \mathrm{S}$ ), es posible indagar el potencial de $T$. aphyllus para generar alteraciones en el fenotipo defensivo expresables a nivel de especie. Una primera aproximación a este conjunto de preguntas consiste en examinar los patrones de variación del fenotipo defensivo multivariado de cada género usando como variable discriminatoria la presencia o ausencia de $T$. aphyllus. El análisis morfométrico se efectuó sobre siete medidas del fenotipo defensivo morfológico del cacto: (1) longitud de espinas apicales, (2) ancho de espinas apicales, (3) longitud de las espinas del perímetro (a $10 \mathrm{~cm}$ bajo el ápice), (4) ancho de las espinas del perímetro, (5) longitud de espinas laterales en la porción medial del cacto, (6) ancho de las espinas en la porción medial del cacto, (7) distancia promedio entre areolas. Al menos 5 ejemplares fueron medidos para cada especie (rango 510). Además de las especies con evidencias de parasitismo y para propósitos comparativos, se incluyó en el análisis las especies no parasitadas por T. aphyllus: Echinopsis atacamensis, Echinopsis deserticola, Echinopsis uelbemanniana, Euychnia iquiquensis y Eulychnia saint-pieana.

Los resultados indican que los dos primeros ejes de variación dieron cuenta del 87,3\% de la varianza para el grupo Echinopsis. Una representación gráfica reveló que las especies parasitadas y no parasitadas se distribuyen de modo continuo en el primer eje de variación (Fig. 5A). No obstante lo anterior, el análisis discriminante sobre los valores propios de los primeros cinco ejes, usando como variable clasificatoria la presencia o ausencia del parásito reveló valores 
estadísticamente significativos (Wilk's $\lambda=0,799$; $\left.\mathrm{F}_{5,387}=19,47 ; \mathrm{P}<0,001\right)$, lo cual indica que la presencia del parásito es suficiente para discriminar el fenotipo defensivo multivariado de las especies. En las especies del género Eulychnia, el análisis de componentes principales para los dos primeros ejes dio cuenta del $96,3 \%$ de la varianza, segregándose notoriamente las especies parasitadas y no parasitadas en torno al primer eje de variación (Fig. 5B). El análisis discriminante reveló que el fenotipo defensivo multivariado tiende a cambiar significativamente en función

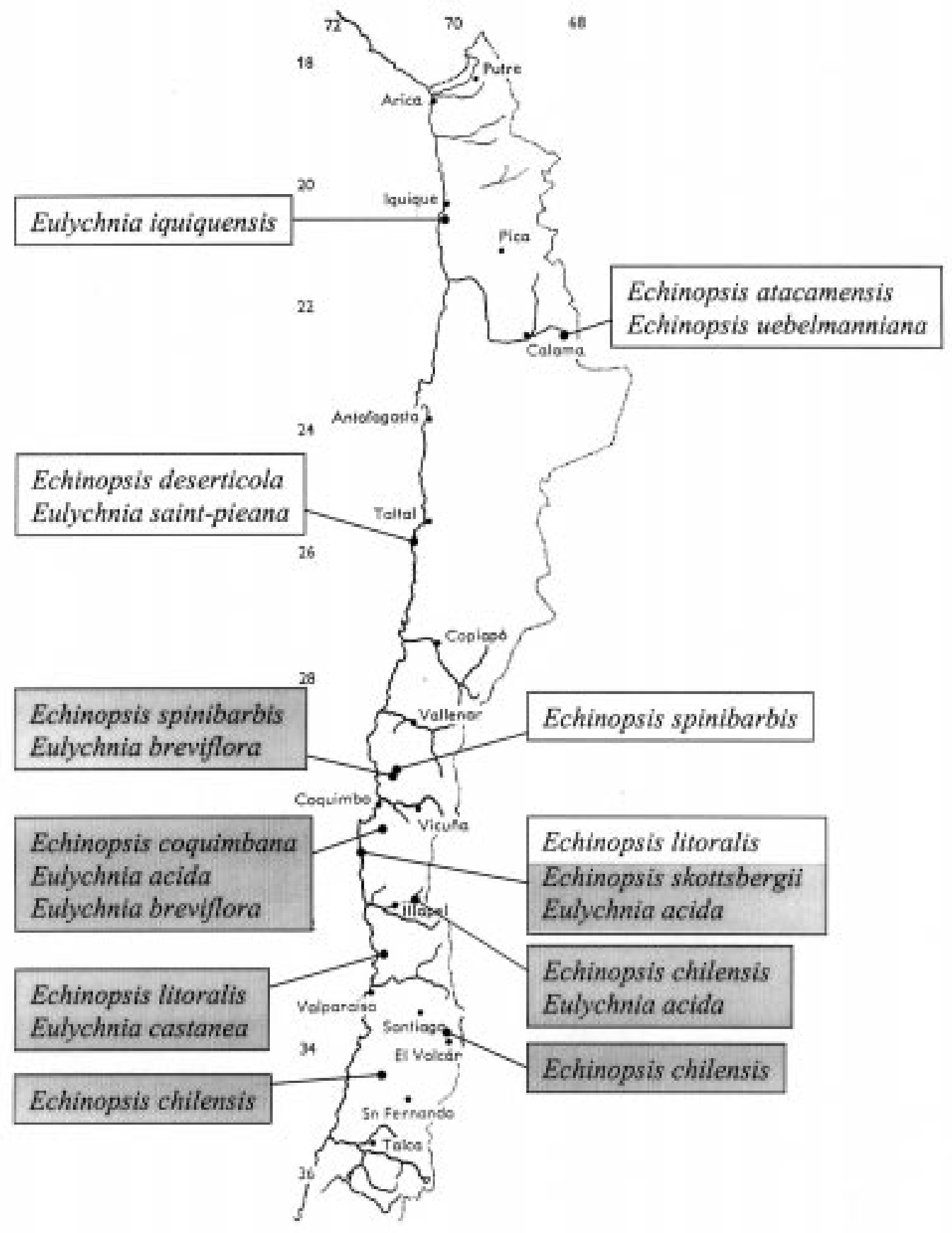

Fig. 4: Combinaciones de especies de cactos parasitados (gris) y no parasitados (blanco) por Tristerix aphyllus en Chile central-norte.

Combinations of coexisting cactus species that are parasitized (grey) and not parasitized (white) by Tristerix aphyllus in north-central Chile. 
del parasitismo (Wilk's $\lambda=0,500 ; \mathrm{F}_{5,201}=40,17$; $\mathrm{P}<0,001)$. Globalmente, los patrones de variación del fenotipo multivariado asociado a las espinas sugieren que tales caracteres pudieron haber cooptado hacia una función defensiva contra la infección por T. aphyllus. Sin embargo, es necesario realizar más estudios que consideren las filogenias particulares para cada género para comprender la trayectoria evolutiva de los fenotipos defensivos en función del parasitismo por T. aphyllus.

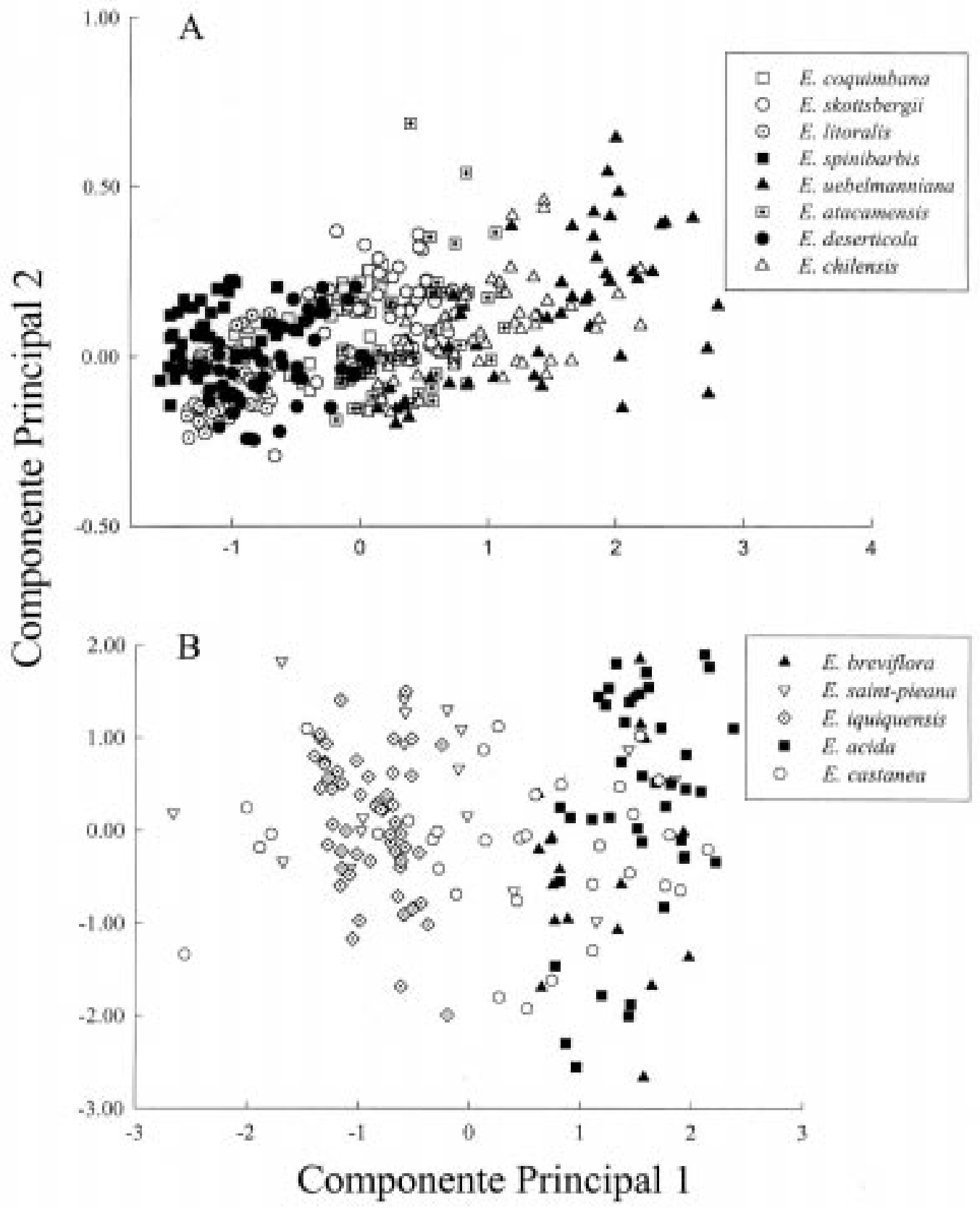

Fig. 5: Representación gráfica de los dos primeros ejes del análisis de componentes principales efectuado sobre siete caracteres morfométricos de las espinas de cactos de los géneros Echinopsis (A) y Eulychnia (B). Las especies parasitadas y no parasitadas por Tristerix aphyllus se muestran en negro y blanco, respectivamente.

Graphic representation of the two first axes of a principal component analysis on seven morphometric spine characters of Echinopsis (A) and Eulychnia (B). The species parasitized and not parasitized by Tristerix aphyllus are depicted in black and white, respectively. 


\section{DISCUSIÓN}

Luego de efectuar una breve pero ilustrativa revisión de los principales temas publicados en la revista "The American Naturalist", Grant (2000) señaló "...evolution is a primary or secondary theme of almost every article, mainly as adaptive explanation for observed phenomena" sugiriendo que esta práctica es uno de los perfiles que mejor caracteriza a los historiadores naturales contemporáneos. Grant continua indicando "The modern naturalist can therefore be characterized as basically an explorer and tester of evolutionary and ecological ideas that are developed to reveal and explain regularities in nature". Los antecedentes de historia natural presentados en este trabajo constituyen la base sine qua non para abordar preguntas acerca del potencial coevolutivo de este singular sistema parásito-hospedero. Aún cuando existe un protocolo de estudio relativamente estandarizado a nivel macroevolutivo para el estudio de la coevolución parásito-hospedero, el cual se basa en el análisis de la congruencia entre las filogenias parásito-hospedero (e.g., Brooks 1988, Brooks \& McLennan 1991, Page 1994), no existe consenso acerca de cómo caracterizar la coevolución parásito-hospedero a un nivel poblacional-microevolutivo (Poulin 1998). Recientemente se ha propuesto que la virulencia es una de las variables que mejor indica el potencial de una interacción parásito-hospedero para coevolucionar, ya que resume tanto la capacidad del parásito para causar daño al hospedero, como la habilidad del hospedero para defenderse del parasitismo. A pesar de algunas discrepancias sobre método e interpretación (e.g., Ebert 1998, Hochberg 1998), la mayor parte de los autores concuerda que la virulencia es un buen indicador de la capacidad de un parásito para matar o reducir la adecuación biológica de su hospedero (e.g., Bull 1994, Frank 1996, Ebert \& Herre 1996, Ebert 1999). Se han explorado al menos teóricamente, una serie de predicciones acerca del curso tomado por la evolución de la virulencia (ver revisiones en Bull 1994, Frank 1996, Ebert \& Herre 1996). Entre tales predicciones hay tres que revisten particular importancia para este sistema. Primero, cepas muy virulentas de un parásito pueden ser mantenidas sólo si el parásito posee una alta capacidad de transmisión (May \& Anderson 1979, Anderson \& May 1982, Ewald 1987, Bull et al. 1991, Herre 1993, Ebert 1994, Herre 1995). Implícita en esta predicción es la correlación entre el nivel de virulencia evolucionado por un parásito y su capacidad de transmisión. Aún cuando este argumento es válido sólo cuando la transmisión ocurre hacia individuos hospederos de la misma generación (transmisión horizontal), excluyendo la transmisión de padres a hijos (transmisión vertical) (Lipsitch et al. 1996), esta relación se ha constituido en un paradigma de los estudios de virulencia. Los antecedentes presentados en este trabajo sugieren que tal evaluación es posible en este sistema. La transmisión es equivalente a la dispersión de semillas, fenómeno tratado extensamente en la literatura y para el cual existen protocolos bien definidos (Martínez del Río et al. 1996). Una estimación de transmisión del muérdago y de la adecuación de los hospederos permitirá indagar sobre la pregunta de la virulencia en este sistema. Segundo, el nivel de virulencia de un parásito depende de la estructura espacial de la población de hospederos. Si la estructura espacial es alta (i.e., si los hospederos susceptibles a la infección se distribuyen agregadamente), la probabilidad de un parásito de reencontarse con un mismo hospedero es alta por lo que su virulencia debiera tender a disminuir a lo largo del tiempo (Lipsitch et al. 1995), pudiendo incluso evolucionar cooperación entre parásitos y hospederos bajo determinadas circunstancias (e.g., Nowak \& May 1994). Tercero, infecciones múltiples sobre un mismo hospedero conllevan a que cepas más agresivas, competitivas y eficientes en la explotación del hospedero sean favorecidas por selección natural, promoviéndose de este modo, fenotipos más virulentos y transmisibles (Frank 1992, Bull 1994, Nowak \& May 1994, May \& Nowak 1995, van Baalen \& Sabelis 1995, Ebert \& Herre 1996, Frank 1996). La base empírica de historia natural del sistema aquí detallado permite abordar el problema de la evolución de la virulencia en cualquiera de los componentes antes mencionados. Por ejemplo, la estructura espacial de los hospederos es fácil de caracterizar mediante técnicas de levantamiento topográfico. Asimismo, la alta tasa de reinfección experimentada por los hospederos individuales (Martínez del Río et al. 1995), permite plantearse la pregunta de la asociación entre infecciones múltiples y virulencia. Actualmente, en nuestra investigación indagamos este tipo de preguntas.

A pesar que la existencia de selección recíproca entre parásitos y hospederos a través de la adquisición de adaptaciones y contra-adaptaciones por selección natural es una condición necesaria para que ocurra coevolución (véase Janzen 1980), muy pocos estudios se han diseñado para cuantificar tal supuesto (véase Davies \& Brooke 1989a, 1989b, Thompson \& Burdon 1992, Clayton et al. 1999). Más aún, los resultados de tales estudios no han producido evidencias inequívocas de selección recíproca local, creando de este modo la 
impresión que la coevolución es poco frecuente en estos sistemas. Una perspectiva de mayor escala para la coevolución entre pares o grupos de especies ha sido propuesta recientemente, la cual ha renovado el interés por los fenómenos coevolutivos (ver suplemento especial en American Naturalist 153: 1999). La teoría del mosaico geográfico propuesta por Thompson (1994, 1999) se basa en estudiar una misma interacción interespecífica a través de múltiples localidades. La teoría tiene la ventaja de incorporar no sólo la dinámica coevolutiva parásito-hospedero a una escala local, que es donde ocurre la selección natural, sino un conjunto inter-relacionado de sistemas locales. De acuerdo a la teoría es posible caracterizar los distintos tipos de efectos selectivos recíprocos entre especies como simétricos, asimétricos, fuertes y débiles, pudiéndose encontrar un rango de posibilidades en los sistemas naturales, desde intensa selección recíproca en algunas localidades ("sitios calientes") hasta una nula selección en otras ("sitios fríos") (Benkman 1999). De acuerdo a la teoría, la heterogeneidad de situaciones locales produciría un mosaico selectivo a nivel del paisaje o sistema metapoblacional, donde las trayectorias evolutivas locales podrían amortiguarse o potenciarse dependiendo del flujo génico, deriva génica, y extinción de las poblaciones de localidades subsidiarias. En último término, la persistencia evolutiva de las interacciones se favorecería a través de flujo génico del parásito, hospedero, o ambos, permitiendo una dinámica coevolutiva espacial y temporalmente estructurada en el sistema metapoblacional. Nosotros abordamos el estudio a mesoescala mediante el examen de los patrones de selección en 10 poblaciones en la Reserva
Nacional Las Chinchillas. Mediante la cuantificación del flujo genético y de los coeficientes de selección, esperamos caracterizar la estructura en mosaico de la interacción coevolutiva. La mayoría de los modelos existentes de coevolución parásito-hospedero presuponen que los parásitos impactan consistentemente en la adecuación darwiniana de los hospederos a lo largo del tiempo y el espacio. Esto no es necesariamente cierto. Información compilada durante 5 años en la Reserva Nacional Las Chinchillas han revelado efectos extremadamente variables del parásito entre distintas poblaciones y a lo largo del tiempo, lo cual indica una relación más intermitente en el tiempo y heterogénea en el espacio que lo concebido inicialmente (Medel 2000). El sistema parásito-hospedero en plantas constituye así un buen sustrato sobre el cual indagar preguntas sobre la naturaleza geográfica de la interacción.

Los caracteres de los hospederos sujetos a selección mediada por parásitos parecieran no sólo expresarse a un nivel poblacional microevolutivo sino también a nivel macroevolutivo. En general, es posible indagar acerca del valor adaptativo de algunos caracteres, al menos mediante dos aproximaciones: (a) poblacional (no histórica) y (b) filogenética (histórica). Aunque existen profundas diferencias tanto conceptuales como metodológicas entre ambas perspectivas (Miles \& Dunham 1993, ver Tabla 4), éstas son complementarias mas que excluyentes, proporcionando distintos puntos de vista a un mismo fenómeno. Mientras la perspectiva poblacional ha sido aplicada en estudios de selección fenotípica de interacciones parásito-hospedero (e.g., Medel 2000, 2001), donde el valor adaptativo de los

TABLA 4

Resumen de características de las perspectivas poblacional y filogenética para el estudio de la adaptación

Summary of characteristics of the population and phylogenetic perspectives for the study of adaptation

\begin{tabular}{lll}
\hline & Perspectiva poblacional & Perspectiva filogenética \\
\hline Foco de análisis & $\begin{array}{l}\text { Examina el efecto de la selección } \\
\text { natural sobre la variación poblacional } \\
\text { y evolución de fenotipos }\end{array}$ & $\begin{array}{l}\text { Examina el efecto de la selección } \\
\text { natural sobre el origen de } \\
\text { caracteres de las especies }\end{array}$ \\
Protocolo & $\begin{array}{l}\text { Explora la relación entre el fenotipo } \\
\text { individual y la adecuación Darwiniana }\end{array}$ & $\begin{array}{l}\text { Explora la evolución de uno o más } \\
\text { caracteres a lo largo de filogenias }\end{array}$ \\
Tipo de evidencias & $\begin{array}{l}\text { Dirección, magnitud y forma de la } \\
\text { selección natural actuando sobre } \\
\text { la población }\end{array}$ & $\begin{array}{l}\text { Independencia, dirección y } \\
\text { magnitud del cambio de caracteres } \\
\text { entre las especies }\end{array}$ \\
Nivel de comparación & Intrapoblacional & Interespecífico \\
\hline
\end{tabular}


caracteres se infiere a través de su asociación con la adecuación Darwiniana de los organismos (véase Lande \& Arnold 1983, Mitchell-Olds \& Shaw 1987, Schluter 1988, Phillips \& Arnold 1989, Schluter \& Nychka 1994), estudios que exploren el valor adaptativo de caracteres cuantitativos de especies involucradas en interacciones parásitohospedero, bajo una perspectiva histórica, son más escasos (pero véase Brooks \& McLennan 1991, Pellmyr 1992) y no exentos de cuestionamiento (e.g., Leroi et al. 1994). El sistema conformado por el muérdago T. aphyllus y sus hospederos cactáceas es apropiado para efectuar análisis de adaptación a ambas escalas puesto que los resultados obtenidos del análisis de componentes principales del fenotipo defensivo entre las especies, es consistente con el valor funcional de los mismos caracteres a escala poblacional. Una etapa necesaria para abordar la pregunta sobre la historia de la asociación es contar con filogenias bien resueltas donde rastrear la trayectoria evolutiva de los caracteres probadamente funcionales en estudios poblacionales.

\section{AGRADECIMIENTOS}

Rodrigo Medel agradece a Anna Traveset y Mary Kalin-Arroyo por su invitación a participar en el Taller "Aspectos Teóricos de la Reproducción en Plantas: consecuencias para la conservación”, financiado por CYTED. La hospitalidad proporcionada por Anna Traveset y sus colaboradores durante el Taller en Palma de Mallorca es igualmente agradecida. Muchas personas han participado en diversas etapas de la adquisición de información. Entre ellos agradecemos especialmente a Jorge Arroyo, Mildred Ehrenfeld y Eric Rivera. Felipe Hinojosa ayudó a comprender el escenario histórico de la interacción. Se agradece a H. Niemeyer por facilitar equipos para la cuantificación de alcaloides. Este trabajo no habría sido posible sin la cooperación permanente de CONAF, IV Región y el personal de la Reserva Nacional Las Chinchillas a cargo de Ignacio Cortés. Rodrigo Medel agradece el financiamiento de DID (Universidad de Chile) y de los proyectos FONDECYT 1940234, 1970497, y 1010660. Carezza Botto agradece el financiamiento de una beca CONICYT para estudios de doctorado y al proyecto FONDECYT 2010022. Marco Méndez agradece el financiamiento del proyecto FONDECYT 3000048. Este trabajo es parte del Centro para Estudios Avanzados en Ecología y Biodiversidad financiado por Milenio P99-103-F ICM. Financiamiento adicional fue proporcionado por una Cátedra Presidencial a Mary Kalin-Arroyo.

\section{LITERATURA CITADA}

ANDERSON RM \& RM MAY (1982) Coevolution of hosts and parasites. Parasitology 85: 411-426.

ALPERS CN \& GH BRIMHALL (1988) Middle Miocene climatic change in the Atacama desert, northern Chile: evidence from supergene mineralization at $\mathrm{La}$ Escondida. Geological Society of America Bulletin 100: 1640-1656.

AMICO G \& MA AIZEN (2000) Mistletoe seed dispersal by a marsupial. Nature 408: 929-930.

ARAYA B \& G MILLIE (1986) Guía de campo de las aves de Chile. Editorial Universitaria, Santiago, Chile. $389 \mathrm{pp}$.

BARLOW BA (1983) Biogeography of Loranthaceae and Viscaceae. En: Calder M \& P Bernhardt (eds) The biology of mistletoes: 19-46. Academic Press, Sydney, Australia.

BENKMAN CW (1999) The selection mosaic and diversifying coevolution between crossbills and lodgepole pine. American Naturalist 153: S75-S91.

BOTTO-MAHAN C, R MEDEL, R GINOCCHIO \& G MONTENEGRO (2000) Factors affecting the circular distribution of the leafless mistletoe Tristerix aphyllus (Loranthaceae) on the cactus Echinopsis chilensis. Revista Chilena de Historia Natural 73: 525-531.

BROOKS DR (1988) Macroevolutionary comparisons of host and parasite phylogenies. Annual Review of Ecology and Systematics 19: 235-259.

BROOKS DR \& DA MCLENNAN (1991) Phylogeny, ecology, and behavior: a research program in comparative biology. University of Chicago Press, Chicago, Illinois.

BULL JJ (1994) Virulence. Evolution 48: 1423-1437.

BULL JJ, I MOLINEUX \& WR RICE (1991) Selection of benevolence in a host-parasite system. Evolution 45: 875-882.

CLAYTON DH, PLM LEE, DM TOMPKINS \& ED BRODIE III (1999) Reciprocal natural selection on host-parasite phenotypes. American Naturalist 154: 261-270.

DAVIES NB \& M DE L BROOKE (1989a) An experimental study of co-evolution between the cuckoo, Cuculus canorus, and its hosts. I. Host egg discrimination. Journal of Animal Ecology 58: 207-224.

DAVIES NB \& M DE L BROOKE (1989b) An experimental study of co-evolution between the cuckoo, Cuculus canorus, and its hosts. II. Host egg markings, chick discrimination and general discussion. Journal of Animal Ecology 58: 225-236.

DE PAMPHILIS CW (1995) Genes and genomes. En: Press MC \& JD Graves (eds) Parasitic plants: 177205. Chapman \& Hall, London, United Kingdom.

DOBSON AP \& ER CRAWLEY (1994) Pathogens and the structure of plant communities. Trends in Ecology and Evolution 9: 393-398.

EBERT D (1994) Virulence and local adaptation of a horizontally transmitted parasite. Science 265: 10841086.

EBERT D (1998) Infectivity, multiple infections, and the genetic correlation between within-host growth and parasite virulence: a reply to Hochberg. Evolution 52: 1869-1871. 
EBERT D (1999) The evolution and expression of parasite virulence. En: Stearns SC (ed) Evolution in health and disease: 161-175. Oxford University Press, Oxford, United Kingdom.

EBERT D \& EA HERRE (1996) The evolution of parasitic diseases. Parasitology Today 12: 96-101.

EWALD PW (1987) Transmission modes and evolution of the parasitism-mutualism continuum. Annals of the New York Academy of Sciences 503: 295-306.

FINEBLUM WL \& MD RAUSHER (1995) Tradeoff between resistance and tolerance to herbivore damage in a morning glory. Nature 377: 517-520.

FOLLMAN G \& M MAHU (1964) Las plantas huéspedes de "Phrygilanthus aphyllus"(Miers) Eichl. Boletín de la Universidad de Chile, Ciencias 7: 39-41.

FRANK SA (1992) A kin selection model for the evolution of virulence. Proceedings of the Royal Society of London, Series B 250: 195-197.

FRANK SA (1996) Models of parasite virulence. Quarterly Review of Biology 71: 37-78.

GOMES AL \& GW FERNANDES (1994) Influence of parasitism by Pilostyles ingae (Rafflesiaceae) on its host plant, Mimosa naguirei (Leguminosae). Annals of Botany 68: 205-208.

GÓMEZ JM (1994) Importance of direct and indirect effects in the interaction between a parasitic angiosperm (Cuscuta epithymum) and its host plant (Hormathophylla spinosa). Oikos 71: 97-106.

GRANT PR (2000) What does it mean to be a naturalist at the end of the twentieth century? American Naturalist 155: 1-12.

HARBORNE JB \& H BAXTER (1993) A handbook of bioactive compounds from plants. Taylor \& Francis, London, United Kingdom.

HARTMANN T (1991) Alkaloids. En: Rosenthal GA \& MR Berenbaum (eds) Herbivores: their interactions with secondary plant metabolites: 79-121. Second edition. Volume I. Academic press, London, United Kingdom.

HERMS DA \& WJ MATTSON (1992) The dilemma of plants: to grow or defend. Quarterly Review of Biology 67: 186-335.

HERRE EA (1993) Population structure and the evolution of virulence in nematode parasites of fig wasps. Science 259: 1442-1445.

HERRE EA (1995) Factors affecting the evolution of virulence: nematode parasites of fig wasps as a case study. Parasitology 111: S179-S191.

HERRERA CM (1988) The fruiting ecology of Osyris quadripartita: individual variation and evolutionary potential. Ecology 69: 233-249.

HOCHBERG ME (1998) Establishing genetic correlations involving parasite virulence. Evolution 52: 1865 1868.

HOFFMANN A (1986) Cactáceas en la flora silvestre de Chile. Ediciones Fundación Claudio Gay, Santiago, Chile. 272 pp.

JANZEN DH (1980) When is it coevolution? Evolution 34: 611-612.
KALIN-ARROYO MT, L CAVIERES, C MARTICORENA \& M MUÑOZ-SCHICK (1995) Convergence in the Mediterranean floras in central Chile and California: insights from comparative biogeography. En: KalinArroyo MT, PH Zedler \& MD Fox (eds) Ecology and biogeography of Mediterranean ecosystems in Chile, California, and Australia: 43-88. Spinger-Verlag, New York, New York.

KUIJT J (1969) The biology of parasitic flowering plants. University of California Press, Berkeley, California. $246 \mathrm{pp}$.

KUIJT J (1988) Revision of Tristerix (Loranthaceae). Systematic Botany Monographs 19: 1-61.

LANDE R \& SJ ARNOLD (1983) The measurement of selection on correlated characters. Evolution 37: 12101226.

LARSON DL (1996) Seed dispersal by specialist versus generalist foragers: the plant's perspective. Oikos 76: 113-120.

LEROI AM, MR ROSE \& GV LAUDER (1994) What does the comparative method reveal about adaptation? American Naturalist 143: 381-402.

LIPSITCH M, EA HERRE \& MA NOWAK (1995) Host population structure and the evolution of virulence: a "law of diminishing returns". Evolution 49: 743-748.

LIPSITCH M, S SILLER \& MA NOWAK (1996) The evolution of virulence in pathogens with vertical and horizontal transmission. Evolution 50: 1729-1741.

MARTÍNEZ DEL RÍO C, M HOURDEQUIN, A SILVA \& R MEDEL (1995) The influence of cactus size and previous infection on bird deposition of mistletoe seeds. Australian Journal of Ecology 20: 571-576.

MARTÍNEZ DEL RÍO C, A SILVA, R MEDEL \& M HOURDEQUIN (1996) Seed dispersers as disease vectors: bird transmission of mistletoe seeds to plant hosts. Ecology 77: 912-921.

MARVIER MA (1996) Parasitic plant-host interactions: plant performance and indirect effects on parasitefeeding herbivores. Ecology 77: 1398-1409.

MATTHIES D (1995) Parasitic and competitive interactions between the hemiparasites Rhinanthus serotinus and Odontites rubra and their host Medicago sativa. Journal of Ecology 83: 245-251.

MATTHIES D (1996) Interactions between the root hemiparasite Melampyrum arvense and mixtures of host plants: heterotrophic benefit and parasitemediated competition. Oikos 75: 118-124.

MAUSETH JD (1985) Relations between Trichocereus chilensis and the holoparasite Tristerix aphyllus. Medio Ambiente (Chile) 7: 39-44.

MAUSETH JD (1991) Botany: an introduction to plant biology. Saunders College Publishing, Philadelphia, Pennsylvania. $800 \mathrm{pp}$.

MAUSETH JD, G MONTENEGRO \& AM WALCKOWIAK (1984) Studies of the holoparasite Tristerix aphyllus (Loranthaceae) infecting Trichocereus chilensis (Cactaceae). Canadian Journal of Botany 62: 847-857.

MAUSETH JD, G MONTENEGRO \& AM WALCKOWIAK (1985) Host infection and flower formation by the parasite Tristerix aphyllus (Loranthaceae). Canadian Journal of Botany 63: 567 581. 
MAY RM \& RM ANDERSON (1979) Population biology of infectious diseases: part II. Nature 280: 455-461.

MAY RM \& MA NOWAK (1995) Coinfection and the evolution of virulence. Proceedings of the Royal Society of London, Series B 261: 209-215.

MEDEL R (2000) Assessment of parasite-mediated selection in a host-parasite system in plants. Ecology 81: 1554-1564.

MEDEL R (2001) Assessment of correlational selection on tolerance and resistance traits in a host plantparasitic plant interaction. Evolutionary Ecology 15 : 37-52.

MEDEL RG, E VERGARA, A SILVA \& IA SEREY (1995) Variation of the architectural phenotype of Tristerix aphyllus in central Chile. Revista Chilena de Historia Natural 68: 451-458.

MILES DB \& AE DUNHAM (1993) Historical perspectives in ecology and evolution. Annual Review of Ecology and Systematics 24: 587-619.

MITCHELL-OLDS T \& RG SHAW (1987) Regression analysis of natural selection: statistical inference and biological interpretation. Evolution 41: 1149-1161.

MURPHY SR, N REID, Z YAN \& WN VENABLES (1993) Differential passage time of mistletoe fruits through the gut of honeyeaters and flowerpeckers: effects on seedling establishment. Oecologia 93: 171-176.

MUSSELMAN LJ \& MC PRESS (1995) Introduction to parasitic plants. En: Press MC \& JD Graves (eds) Parasitic plants: 1-13. Chapman and Hall, London, United Kingdom.

MUTIKAINEN P, V SALONEN, S PUUSTINEN \& $\mathrm{T}$ KOSKELA (2000) Local adaptation, resistance, and virulence in a hemiparasitic plant-host plant interaction. Evolution 54: 433-440.

NORTON DA \& MA CARPENTER (1998) Mistletoes as parasites: host specificity and speciation. Trends in Ecology and Evolution 13: 101-105.

NOWAK MA \& RM MAY (1994) Superinfection and the evolution of parasite virulence. Proceedings of the Royal Society of London, Series B 255: 81-89.

PAGE RDM (1994) Parallel phylogenies: reconstructing the history of host-parasite assemblages. Cladistics 10: $155-173$.

PELLMYR O (1992) The phylogeny of a mutualism: evolution and coadaptation between Trollius and its seed parasitic pollinators. Biological Journal of the Linnean Society 47: 337-365.

PHILLIPS PC \& SJ ARNOLD (1989) Visualizing multivariate selection. Evolution 43: 1209-1222.

POULIN R (1998) Evolutionary ecology of parasites: from individuals to communities. Chapman \& Hall, London, United Kingdom. 212 pp.

REID N (1986) Pollination and seed dispersal of mistletoes (Loranthaceae) by birds in southern Australia. En: Ford HA \& DC Paton (eds) The dynamic partnership: birds and plants in southern Australia: 61-91. Govt Printer, Adelaide, Australia.
REID N (1989) Dispersal of mistletoes by honeyeaters and flowerpeckers: components of seed dispersal quality. Ecology 70: 137-145.

REID N (1991) Coevolution of mistletoe and frugivorous birds? Australian Journal of Ecology 16: 457-469.

ROSENTHAL GA \& MR BERENBAUM (1991) Herbivores: their interactions with secondary plant metabolites. Second edition. Volume I. Academic press, London, United Kingdom. 468 pp.

RUNDEL PW (1976) Population variability in the genus Trichocereus (Cactaceae) in central Chile. Plant Systematics and Evolution 127: 1-9.

SCHLUTER D (1988) Estimating the form of natural selection on a quantitative trait. Evolution 42: 849861.

SCHLUTER D \& D NYCHKA (1994) Exploring fitness surfaces. American Naturalist 143: 597-616.

SILVA A \& C MARTÍNEZ DEL RÍO (1996) Effects of the mistletoe Tristerix aphyllus (Loranthaceae) on the reproduction of its cactus host Echinopsis chilensis. Oikos 75: 437-442.

SMITH-RAMÍREZ C (1999) Selección fenotípica secuencial sobre rasgos reproductivos del muérdago Tristerix aphyllus. Tesis de Doctorado. Facultad de Ciencias, Universidad de Chile. Santiago, Chile. 87 pp.

SOLBRIG O (1976) The origin and floristic affinities of the South American temperate desert and semidesert regions. En: Goodall W (ed) Evolution of desert biota: 7-49. University of Texas Press, Austin, Texas.

SOLBRIG O, ML CODY, ER FUENTES, W GLANZ, JH HUNT \& AR MOLDENKE (1977) The origin of the biota. En: Mooney HA (ed) Convergent evolution in Chile and California: Mediterranean climate ecosystems: 13-26. Dowden, Hutchinson \& Ross, Stroudsburg. Pennsylvania.

THOMPSON JN (1994) The coevolutionary process. University of Chicago Press, Chicago, Illinois. 376 pp.

THOMPSON JN (1999) Specific hypotheses on the geographic mosaic of coevolution. American Naturalist 153: S1-S14.

THOMPSON JN \& JJ BURDON (1992) Gene-for-gene coevolution between plants and parasites. Nature 360: 121-125.

VAN BAALEN M \& MW SABELIS (1995) The dynamics of multiple infection and the evolution of virulence. American Naturalist 146: 881-910.

VILLAGRÁN C \& LF HINOJOSA (1997) Historia de los bosques del sur de Sudamérica, II: análisis fitogeográfico. Revista Chilena de Historia Natural 70: 241-267.

WATKINSON AR \& CC GIBSON (1988) Plant parasitism: the population dynamics of parasitic plants and their effects upon community structure. En: Davy AJ, MJ Hutchings \& AR Watkinson (eds) Plant population ecology: 393-411. Blackwell Scientific Publications, Oxford, United Kingdom. 